\title{
GH dysfunction in Engrailed-2 knockout mice, a model for autism spectrum disorders
}

\section{Giovanni Provenzano ${ }^{1 \neq}$, Elena Clementi ${ }^{1 \neq}$, Sacha Genovesi ${ }^{1}$, Manuela Scali ${ }^{2,3}$, Prem Prakash Tripathi ${ }^{2,3+}$, Paola Sgadò ${ }^{1}$ and Yuri Bozzi ${ }^{1,2 *}$}

1 Laboratory of Molecular Neuropathology, Centre for Integrative Biology (CIBIO), University of Trento, Trento, Italy

${ }^{2}$ Neuroscience Institute, National Research Council (CNR), Pisa, Italy

${ }^{3}$ Laboratory of Neurobiology, Scuola Normale Superiore, Pisa, Italy

\section{Edited by:}

Roberto Canitano, University Hospital of Siena, Italy

Reviewed by:

Munis Dundar, Erciyes University, Turkey

Alessandro Sale, National Research Council (CNR), Italy

Anne Leighton Calof, University of California, Irvine, USA

\section{*Correspondence:}

Yuri Bozzi, Laboratory of Molecular Neuropathology, Centre for

Integrative Biology (CIBIO), University of Trento, Via delle Regole 101,

Mattarello, Trento 38123, Italy

e-mail: bozzi@science.unitn.it

\section{${ }^{\dagger}$ Present address:}

Prem Prakash Tripathi, Seattle

Children's Research Institute, Seattle, WA, USA

${ }^{\ddagger}$ Giovanni Provenzano and Elena Clementi have contributed equally to this work.
Insulin-like growth factor 1 (IGF-1) signaling promotes brain development and plasticity. Altered IGF-1 expression has been associated to autism spectrum disorders (ASD). IGF-1 levels were found increased in the blood and decreased in the cerebrospinal fluid of ASD children. Accordingly, IGF-1 treatment can rescue behavioral deficits in mouse models of ASD, and IGF-1 trials have been proposed for ASD children. IGF-1 is mainly synthesized in the liver, and its synthesis is dependent on growth hormone $(\mathrm{GH})$ produced in the pituitary gland. GH also modulates cognitive functions, and altered levels of $\mathrm{GH}$ have been detected in ASD patients. Here, we analyzed the expression of GH, IGF-1, their receptors, and regulatory hormones in the neuroendocrine system of adult male mice

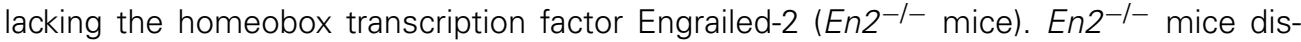
play ASD-like behaviors (social interactions, defective spatial learning, increased seizure susceptibility) accompanied by relevant neuropathological changes (loss of cerebellar and forebrain inhibitory neurons). Recent studies showed that En2 modulates IGF-1 activity during postnatal cerebellar development. We found that GH mRNA expression was markedly deregulated throughout the neuroendocrine axis in En2 ${ }^{-1-}$ mice, as compared to wildtype controls. In mutant mice, GH mRNA levels were significantly increased in the pituitary gland, blood, and liver, whereas decreased levels were detected in the hippocampus. These changes were paralleled by decreased levels of $\mathrm{GH}$ protein in the hippocampus but not other tissues of En2 ${ }^{-/-}$mice. IGF-1 mRNA was significantly up-regulated in the liver and down-regulated in the En2 $2^{-I-}$ hippocampus, but no differences were detected in the levels of IGF-1 protein between the two genotypes. Our data strengthen the notion that altered $\mathrm{GH}$ levels in the hippocampus may be involved in learning disabilities associated to ASD.

Keywords: autism spectrum disorders, hippocampus, pituitary gland, liver, growth hormone, insulin-like growth factor, neuroendocrine axis, mouse model

\section{INTRODUCTION}

Insulin-like growth factor 1 (IGF-1) is a hormone primarily produced by the liver, which exerts an endocrine action on multiple target tissues. It is also locally produced in tissues, including brain, where it acts in a paracrine/autocrine fashion. Several studies demonstrate that IGF-1 profoundly modulates brain function, both during development and adult life. During development, IGF-1 promotes neuronal survival [reviewed in Ref. (1)] and maturation of cortical and retinal function (2-4), whereas in adult life, it exerts multiple actions ranging from the control of synaptic plasticity to neuroprotection [reviewed in Ref. $(1,5)]$. During adulthood, IGF-1 is crucial for both basal and exercise-induced hippocampal neurogenesis $(6,7)$ and markedly regulates learning and cognition (8).

A link between IGF-1 pathway and autism spectrum disorders (ASD) has been proposed. IGF-1 levels have been found increased in the blood (9) and decreased in the cerebrospinal fluid $(10,11)$ of ASD children, as compared to healthy individuals. In addition, deregulation of IGF-1 signaling pathway, which involves downstream effectors such as PI3 kinase (PI3K), protein kinase B (AKT), and mammalian target of rapamycin (mTOR), has been shown in syndromic forms of ASD [reviewed in Ref. (12)]. This led to propose that IGF-1 treatment might be beneficial for ASD, and clinical trials have been approved ${ }^{1}$. Indeed, IGF-1 administration is able to rescue ASD-related molecular changes in neurons derived from ASD patients (13) as well as ASD-like behavioral deficits in mouse models $(14,15)$. Altered levels of other hormones of the IGF-1 pathway have been associated to ASD (9). IGF-1 synthesis in the liver depends on levels of circulating growth hormone (GH) produced in the pituitary gland. GH also modulates cognitive functions (16), and altered GH levels have been detected in ASD patients (17).

Genome-wide association studies identified the transcription factor Engrailed-2 (En2) as a candidate gene for ASD (18), and two recent studies confirmed that En2 expression is altered in the

\footnotetext{
${ }^{1} \mathrm{http}: / /$ clinicaltrials.gov
} 
cerebellum of ASD patients (19,20). Mice lacking the homeobox domain of En2 [En2 $2^{h d / h d}$ mice; (21); here referred to as $E n 2^{-/-}$] are considered a suitable animal model to study the neurodevelopmental basis of ASD. En2 $2^{-/-}$mice display neuropathological and behavioral changes relevant to ASD. Reduced social interactions, defective spatial learning (22-24), and increased seizure susceptibility (25) accompanied by neuropathological changes relevant to ASD [loss of cerebellar and forebrain inhibitory neurons; $(26,27)]$ were indeed described in En $2^{-l-}$ mice. In addition, we recently showed that several genes related to ASD are markedly deregulated in the cerebellum and hippocampus of En2 $2^{-1-}$ mice (28), thus indicating that En2 mutants are a reliable model to investigate gene expression changes relevant to ASD.

A recent study established an important link between En2 and the modulation of IGF-1 pathway (29). During postnatal development, En2 controls proliferation and differentiation of cerebellar granule neuron precursors (GNPs). Notably, in postnatal $E n 2^{-1-}$ cerebellum, the activity of downstream effectors of IGF-1 is increased, and IGF-1 has a stronger mitogenic effect on GNPs, as compared to WT (29). These results indicate that En2 negatively regulates IGF-1 signaling during postnatal cerebellar development.

Here, we investigated the relationship between En2 and GH/IGF-1 pathway in the neuroendocrine system. We analyzed the expression of GH, IGF-1, their receptors, and regulatory hormones in the brain-pituitary-liver axis of adult wild-type (WT) and $E n 2^{-I-}$ mice. We show that GH levels are lowered in the hippocampus of $E n 2^{-1-}$ mice, suggesting that this alteration might contribute to learning disabilities in this ASD mouse model.

\section{MATERIALS AND METHODS \\ ANIMALS}

Experiments were conducted in conformity with the European Community Directive 2010/63/EU and were approved by the Italian Ministry of Health. Animals were housed in a 12-h light/dark cycle with food and water available ad libitum, and all efforts were made to minimize animal suffering during the experiments. The generation of En2 mutants were originally generated on a mixed $129 \mathrm{~Sv} \times \mathrm{C} 57 \mathrm{BL} / 6$ genetic background (21) and then backcrossed at least five times into a C57BL/6 background (27). En $2^{+/+}$(WT) and $E n 2^{-1-}$ mice used in this study were obtained by heterozygous mating $\left(E n 2^{ \pm} \times E n 2^{ \pm}\right)$and genotyped by PCR as previously described (27). A total of 34 adult (3-5 months old) male mice were used: 6 mice per genotype for quantitative RT-PCR, 5 mice per genotype for enzyme-linked immuno-sorbent assay (ELISA) tests, 3 mice per genotype for immunohistochemistry, and 3 mice per genotype for in situ hybridization.

\section{QUANTITATIVE RT-PCR}

Tissues from WT and $E n 2^{-1-}$ mice ( $n=6$ per genotype) were dissected and frozen in dry ice. Blood samples were centrifuged in an Eppendorf benchtop centrifuge for $15 \mathrm{~min}$ at 3,000 rpm to separate serum from cell fraction, and then frozen in dry ice. All samples were stored at $-80^{\circ} \mathrm{C}$ until use. Total RNAs were extracted by Trizol reagent (Invitrogen), treated with DNAse, purified by the RNAeasy Kit (Qiagen), and pooled. cDNAs were synthesized from pooled RNAs $(3 \mu \mathrm{g})$ by SuperScript VILO cDNA Synthesis Kit (Invitrogen). Quantitative reverse-transcription PCR (RT-PCR) was performed in a $\mathrm{C} 1000$ thermal cycler (BioRad) with realtime detection of fluorescence, using the KAPA SYBR FAST master mix reagent (Resnova). Mouse mitochondrial ribosomal protein L41 (Mrpl41) was used as a standard for quantification. Primers (Sigma Genosys, UK) sequences are reported in Table 1. Ratios of comparative concentrations of each mRNA with respect to L41 mRNA were then calculated and plotted as the average of three to four independent reactions with technical replicates obtained from each RNA pool. Expression analyses were performed using the CFX3 Manager (BioRad) software.

\section{BIOINFORMATIC ANALYSIS}

Analysis of En2 binding sites on GH, GHR, mGRF, SST, IGF-1, and IGF-1R gene promoters was performed using the Matinspector web-based search algorithm available from Genomatix Software ${ }^{2}$. The algorithm calculated the similarity between the core motif and overall sequence of the En 2 consensus binding site (matrix ID: $\mathrm{V} \$ E N 2.01)$ and the gene promoter sequences of interest. The En2 matrix was determined based on the Genomatix Matrix Library Version 9.1.

\section{IMMUNOHISTOCHEMISTRY}

Brains from adult WT and $E n 2^{-1-}$ mice ( $n=3$ per genotype) were used for immunohistochemical characterization of

\footnotetext{
${ }^{2}$ http://www.Genomatix.de
}

Table 1 | Primers used for quantitative RT-PCR experiments.

\begin{tabular}{|c|c|c|c|}
\hline Gene & GenBank no. & Forward primer $\left(5^{\prime}-3^{\prime}\right)$ & Reverse primer $\left(5^{\prime}-3^{\prime}\right)$ \\
\hline En2 & NM_010134.3 & ACTGCACGCGCTATTCTG & ACCTGTTGGTCTGAAACTCAG \\
\hline $\mathrm{GH}$ & NM_008117.3 & GCAATGGCTACAGACTCT & AAACAGACTGGACAAGGG \\
\hline IGF- 1 class 1 & NM_001111275.1 & AGCGATGGGGAAAATCAGCA & CAGAGCGCCAGGTAGAAGAG \\
\hline IGF-1 class 2 & NM_001111276.1 & CTGATGTCTGGTCCTTCGGG & CACCСTCCATGACGAAACGA \\
\hline SST & NM_009215 & AGGACGAGATGAGGCTGG & CAGGAGTTAAGGAAGAGATATGGG \\
\hline
\end{tabular}

Abbreviations are as in the text. 
hypothalamic SST. Brains were fixed by transcardial perfusion with $4 \%$ paraformaldehyde followed by $1 \mathrm{~h}$ post-fixation, and coronal sections ( $40 \mu \mathrm{m}$ thickness) were cut by a vibratome (Leica). Serial sections at level of the hypothalamus were incubated overnight with and anti-SST rabbit polyclonal (Peninsula-Bachem; 1:2000 dilution). Signals were revealed using appropriate secondary antibodies and fluorophores as described (27). Hypothalamic nuclei were identified according to the Allen Brain Atlas ${ }^{3}$. Images were acquired at $10 \times$ objective magnification using a Zeiss AxioImager M2 microscope.

\section{IN SITU HYBRIDIZATION}

Brains from WT and $E n 2^{-1-}$ mice ( $n=3$ per genotype) were rapidly removed and frozen on dry ice. Coronal cryostat sections ( $20 \mu \mathrm{m}$ thick) were fixed in $4 \%$ paraformaldehyde. Nonradioactive in situ hybridization was performed as previously described (27) using digoxigenin-labeled riboprobes specific for GH [GenBank ID: X02891; (30)], mGRF (31), and IGF-1 (GenBank ID: NM_010512). The IGF-1 cDNA was cloned by PCR from hippocampal cDNA and recognizes both class 1 and class 2 IGF-1 mRNAs. Signal was detected by alkaline phosphatase-conjugated anti-digoxigenin antibody followed by alkaline phosphatase staining. The specificity of the results was confirmed by the use of sense riboprobes (not shown).

\section{ENZYME-LINKED IMMUNO-SORBENT ASSAY}

Blood samples ( $n=5$ per genotype) were collected at the time of animal sacrifice, kept in ice for $30 \mathrm{~min}$, and then centrifuged in an Eppendorf benchtop centrifuge for $15 \mathrm{~min}$ at 3,000 rpm to separate serum. Dissected liver and hippocampal tissues $(n=5$ per genotype) were homogenized in lysis buffer $(50 \mathrm{mM}$ Tris- $\mathrm{HCl} \mathrm{pH}$ 7.5, 1\% NP-40, 1\% Triton-100, 1 mM PMSF, 10\% glycerol, and protease inhibitor cocktail; Sigma-Aldrich). Homogenates were incubated in ice for $30 \mathrm{~min}$, centrifuged at $12,000 \mathrm{rpm}$ for $5 \mathrm{~min}$ at $4^{\circ} \mathrm{C}$, and supernatants were recovered and stored at $-80^{\circ} \mathrm{C}$. Protein concentration in serum, liver, and hippocampal samples was determined by BCA method (Pierce). Samples were processed for GH (EZRMGH-45K, Millipore) and IGF-1 (ELM-IGFI; RayBiotech) ELISA according to manufacturers' protocol. All samples were analyzed in duplicate.

\section{STATISTICAL ANALYSIS}

Statistical analysis was performed with SigmaPlot 11.0 and Prism 6 (GraphPad) softwares. Values were expressed as mean \pm SEM and quantitative gene expression differences between WT and En2 ${ }^{-1-}$ mice were assessed by Student's $t$-test, with the level of statistical significance set at $p<0.05$.

\section{RESULTS \\ EXPRESSION OF En2 AND IGF-1 SIGNALING GENES IN THE MOUSE BRAIN-PITUITARY-LIVER AXIS}

By using quantitative RT-PCR, we first investigated mRNA expression of En2, GH, GH receptor (GHR), mGRF, SST, IGF-1, and IGF-1 receptor (IGF-1R) in the neuroendocrine axis of WT adult

${ }^{3}$ http://www.brain-map.org/ male mice from our colony. mRNA expression was studied in the hypothalamus, pituitary gland, and liver (the crucial tissues involved in GH and IGF-1 synthesis), as well as hippocampus and blood cell fraction. In agreement with previous findings, we confirmed that En2 mRNA is expressed in the hypothalamus and hippocampus $(23,25,27)$. En2 mRNA was also expressed at detectable levels in blood, pituitary gland, and liver (Figure 1A). As expected, GH mRNA was mainly detected in the pituitary gland, while much lower levels were present in the hippocampus, hypothalamus, liver, and blood (Figure 1B). Consistent with the notion that liver is the main target of GH action, we found GHR mRNA predominantly expressed in the liver, and at lower levels in the other tissues analyzed (Figure 1C). mRNA expression of mGRF and SST, the two hypothalamic hormones controlling GH synthesis, was mainly detected in the hypothalamus (Figures 1D,E). High levels of SST mRNA were also present in the hippocampus, as previously described (27). Two major different transcripts have been described for IGF-1 [class 1 and class 2; $(32,33)$ ]. Both IGF1 class 1 and class 2 mRNAs were predominantly expressed in the liver (Figures 1F,G), as expected (32). Finally, IGF-1R mRNA was mainly expressed in the pituitary gland (the target for IGF1 negative feedback for $\mathrm{GH}$ production) (Figure $\mathbf{1 H}$ ), but also throughout the neuroendocrine axis, consistent with the widespread action of IGF-1 on multiple tissues. These results clearly indicate that our RT-PCR protocol can detect the expression of genes belonging to the GH/IGF-1 pathway in the appropriate tissues throughout the brain-pituitary-liver axis.

\section{ALTERED GH mRNA EXPRESSION IN THE BRAIN-PITUITARY-LIVER AXIS OF En2-I- MICE}

Before investigating GH, GHR, mGRF, SST, IGF-1, and IGF-1R mRNA expression in the neuroendocrine axis of $E n 2^{-1-}$ mice, we first verified whether En2 might directly regulate their transcription. Indeed, bioinformatic analysis revealed that En 2 binding sites are present in the promoters of all these genes (Table 2). We next studied GH and GHR mRNA expression in the neuroendocrine axis of WT and En $2^{-l-}$ adult mice. We found that GH mRNA expression was markedly deregulated throughout the neuroendocrine axis in En2 $2^{-1-}$ mice. A statistically significant increase of GH mRNA levels was detected in the pituitary gland $(+72 \%, p<0.001)$, liver $(+376 \%, p<0.001)$, and blood $(+87 \%, p<0.05)$ of $E n 2^{-l-}$ mice, as compared to WT littermates (Figure 2A). GH mRNA levels were instead significantly lower in the $E n 2^{-l-}$ hypothalamus $(-97 \%, p<0.001)$ and hippocampus ( $-98 \%, p<0.001)$, as compared to WT (Figure $2 \mathrm{~A}$ ). In situ hybridization confirmed GH mRNA decrease in the En2 $2^{-1-}$ hippocampus, mainly in the CA3 subfield (Figure 2C). No significant differences in GHR mRNA levels were detected between genotypes in the analyzed tissues, with the exception of blood, where a marked increase was detected in mutant mice compared to controls $(+312 \%, p<0.05)$ (Figure 2B).

\section{ALTERED EXPRESSION OF MGRF AND SST IN THE HYPOTHALAMUS OF En2 $^{-1-}$ MICE}

We then investigated the expression of mGRF (also known as growth hormone releasing hormone, GHRH) and SST, the two hypothalamic hormones regulating GH synthesis, in the 

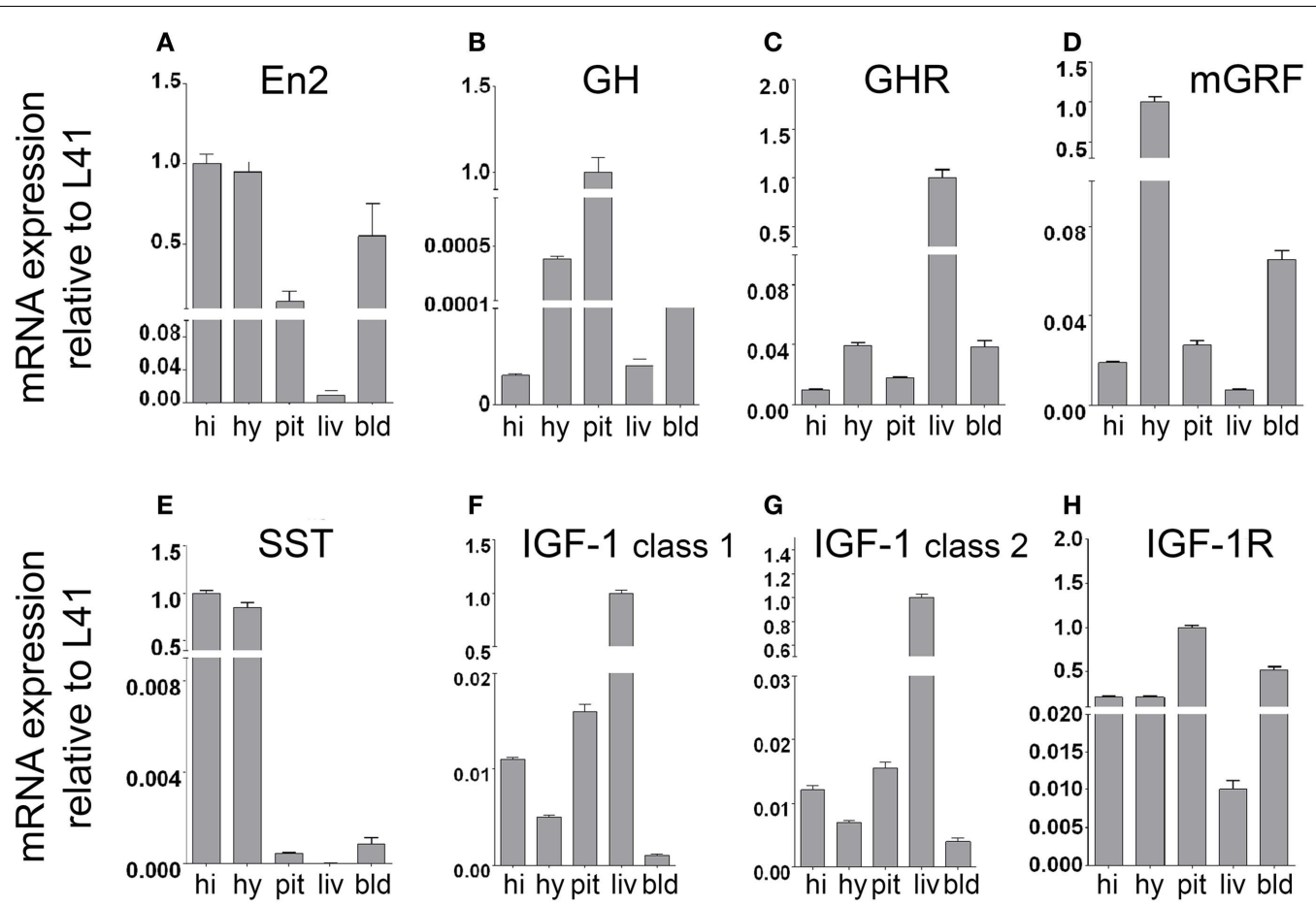

FIGURE 1 | mRNA expression of En2 and genes involved in the GH/IGF-1 pathway in the neuroendocrine axis of WT mice. En2 (A), GH (B), GHR (C), mGRF (D), SST (E), IGF-1 (F,G), and IGF-1R (H) mRNA expression levels in the hippocampus, hypothalamus, pituitary gland, liver, and blood, obtained by quantitative RT-PCR. For each mRNA, relative expression levels (normalized to L41) are reported on a log scale. Two different transcripts (class 1 and class 2) were analyzed for IGF-1. Values are plotted as mean \pm SEM of three independent experiments. Abbreviations: hi, hippocampus; hy, hypothalamus; pit, pituitary gland; liv, liver; bld, blood (cell fraction). Other abbreviations are as in the text.

Table 2 | Presence of En2 binding sites onto GH, GHR, mGRF, SST, IGF-1, and IGF-1R gene promoters.

\begin{tabular}{lllccccc}
\hline Gene & Accession no. & Start & End & Strand & Core & Matrix & Sequence \\
\hline GH & GXP_219787 & 534 & 552 & + & 0.752 & 0.73 & agccatgAATAaatgtata \\
GHR & GXP_889069 & 584 & 602 & - & 1 & 0.863 & ccccataAATTaataatcc \\
IGF-1 & GXP_4345839 & 966 & 984 & - & 1 & 0.858 & tttatgAATTaagccctc \\
IGF-1R & GXP_183481 & 512 & 530 & - & 1 & 0.854 & aacattgAATTagttcttg \\
mGRF & GXP_4357410 & 266 & 284 & - & 1 & 0.797 & ggaaacaAATTgaacaaat \\
SST & GXP_82140 & 62 & 80 & - & 1 & 0.774 & cagaatgAATTtgcaatta \\
\hline
\end{tabular}

The promoter regions of the six genes belonging to the GH/IGF-1 pathway were analyzed for the presence of En2 binding site using the Matinspector program. Core similarity (Core) indicates the match against the nucleotides representing the core motif of the En2 binding site (AATT), where 1 is 100\% identity. Matrix similarity (Matrix) is the overall similarity to the En2 binding motif, where 1 is $100 \%$ identity. For each sequence, the start/end position respect to ATG is indicated, together with the strand on which they are present. For each promoter, several regions containing the En2 binding site were found; the table reports only those with highest values for core and matrix similarity. Genomatix Promoter database (GXP) accession numbers are given for each gene. Abbreviations are as in the text.

brain-pituitary-liver axis of WT and $E n 2^{-1-}$ adult mice. As compared to WT controls, a marked increase of mGRF mRNA ( $+53 \%$, $p<0.05$; Figure 3A) and a significant decrease of SST mRNA $(-13 \%, p<0.01$; Figure 3B $)$ was found in the $E n 2^{-/-}$hypothalamus. Significantly higher mRNA levels for the two hormones were also found in blood (mGRF: $+125 \%, p<0.05$ ) and liver (mGRF: $+129 \%, p<0.01$; SST: $+147 \%, p<0.001)$ from $E n 2^{-/-}$mice (Figures 3A,B). According to our previous study (27), lower levels of SST mRNA were found in the En2 ${ }^{-l-}$ hippocampus, as compared to WT $(-10 \%, p<0.05$; Figure 3B). In situ hybridization and immunohistochemistry experiments, respectively, confirmed the increased expression of mGRF mRNA and decreased levels of SST protein in the dorsomedial/ventromedial paraventricular nuclei of the $\mathrm{En}^{-1-}$ hypothalamus, as compared to WT (Figure 3C).

\section{ALTERED IGF-1 mRNA EXPRESSION IN THE BRAIN-PITUITARY-LIVER AXIS OF En2 ${ }^{-I-}$ MICE}

We next analyzed mRNA expression of IGF-1 and its receptor in the brain-pituitary-liver axis of WT and $E n 2^{-1-}$ adult mice. 


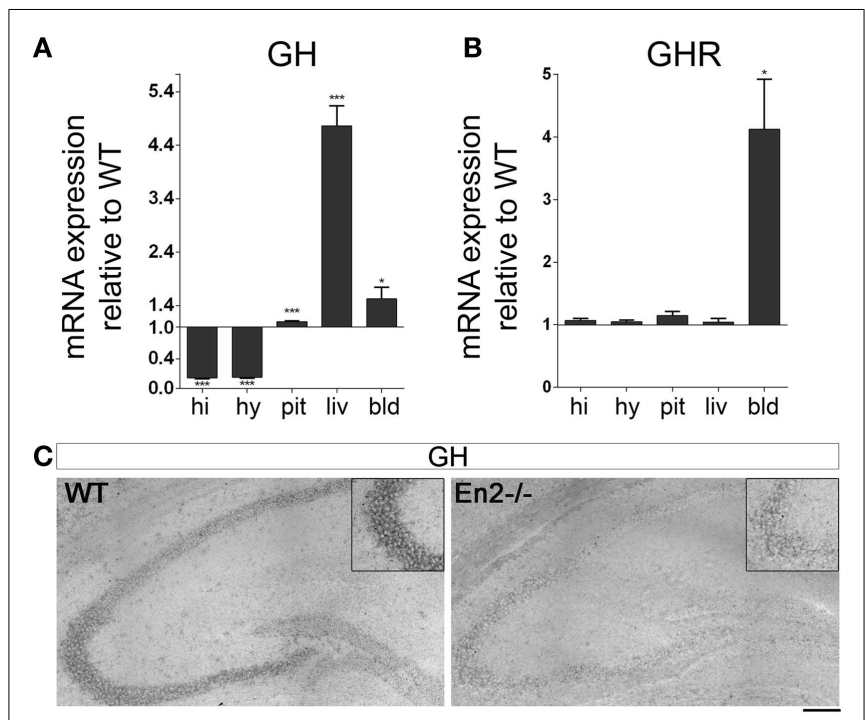

FIGURE 2 | Expression of GH and GH receptor mRNAs in the neuroendocrine axis of WT and $E_{n 2^{-/-}}$mice. (A) GH mRNA quantitative RT-PCR. (B) GHR quantitative RT-PCR. Values are plotted as each gene/L41 comparative quantitation ratios normalized on the expression of WT (mean \pm SEM of three replicates from pools of six animals per genotype; ${ }^{*} p<0.05,{ }^{* *} p<0.001$; Student's $t$-test, En2 ${ }^{-1-}$ vs. WT).

(C) Representative pictures of GH mRNA in situ hybridization on the dorsal hippocampus from WT and En2 $2^{-l-}$ mice. Insets show the CA3 subfield. Scale bar: $200 \mu \mathrm{m}$ (whole hippocampi) and $125 \mu \mathrm{m}$ (insets). Abbreviations are as in Figure 1.

IGF-1 mRNA exists in two major forms (class 1 and class 2), class 2 mRNA transcription being directly regulated by GH (33, 34). We found that levels of IGF-1 class 1 mRNA were significantly reduced in the hippocampus but not other tissues of En $2^{-I-}$ mice, as compared to WT $(-18 \%, p<0.05$ for all comparisons) (Figure 4A). In keeping with GH mRNA expression data (Figure 2), IGF-1 class 2 mRNA was significantly up-regulated in the liver $(+43 \%, p<0.001)$ and down-regulated in the hypothalamus $(-19 \%, p<0.001)$ of $E n 2^{-1-}$ mice, as compared to WT littermates; increased levels of IGF-1 class 2 mRNA were also detected in $E n 2^{-1-}$ pituitary gland $(+16 \%, p<0.01)$ and blood $(+312 \%, p<0.001)$ (Figure 4B). In situ hybridization with a riboprobe specific for both class 1 and class 2 IGF- 1 mRNAs confirmed that IGF-1 mRNA levels are decreased in the $E n 2^{-1-}$ hippocampus (Figure 4D). Finally, IGF-1R mRNA levels did not differ between genotypes in hypothalamus, hippocampus, and pituitary gland, while a significant increase was found in blood $(+84 \%, p<0.01)$ and liver $(+52 \%, p<0.05)$ from $E n 2^{-1-}$ mice compared to WT controls (Figure 4C). Table 3 summarizes mRNA expression data for all the analyzed genes in the brain-pituitary-liver axis of WT and $E n 2^{-1-}$ adult mice.

\section{REDUCED LEVELS OF GH PROTEIN IN THE En2-I- HIPPOCAMPUS}

The altered levels of GH and IGF-1 mRNA expression detected in the $E n 2^{-/-}$neuroendocrine axis prompted us to investigate the levels of these hormones in the hippocampus, serum, and liver of both genotypes. ELISA assays revealed a significant reduction $(-54 \%, p<0.05)$ of $\mathrm{GH}$ protein levels in the hippocampus of

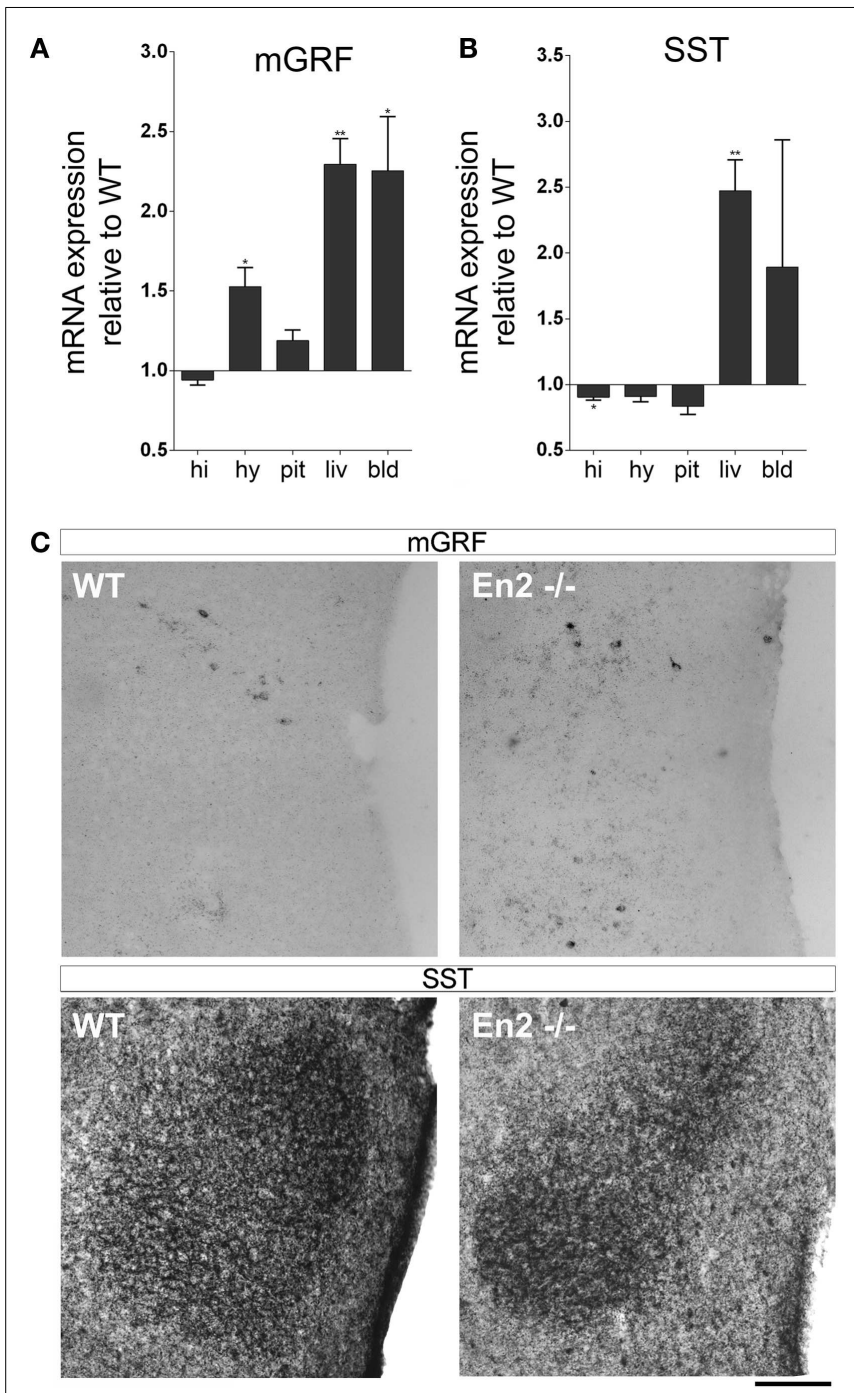

FIGURE 3 | Expression of $m$ GRF and SST $m R N A s$ in the neuroendocrine axis of WT and $E_{n 2^{-1-}}$ mice. (A,B) mRNA expression levels of mGRF (A) and SST (B), obtained by quantitative RT-PCR. Values are plotted as each gene/L41 comparative quantitation ratios normalized on the expression of WT (mean \pm SEM of three replicates from pools of six animals per genotype; ${ }^{*} p<0.05,{ }^{*} p<0.01$; Student's $t$-test, En2 ${ }^{-1-}$ vs. WT). (C) mGRF in situ hybridization and SST immunohistochemistry. Representative pictures show mGRF mRNA and SST protein staining in the dorsomedial and ventromedial paraventricular nuclei of the hypothalamus. Scale bar: $200 \mu \mathrm{m}$. Abbreviations are as in Figure 1.

En $2^{-1-}$ mice, as compared to WT littermates, while no difference was detected in serum samples (Figure 5A). Hippocampal, liver, and serum IGF-1 protein levels did not significantly differ between the two genotypes (Figure 5B).

\section{DISCUSSION}

\section{BRIEF SUMMARY OF RESULTS}

In this study, we analyzed the expression of GH, IGF-1, their receptors, and regulatory hormones in the brain-pituitary-liver axis of adult En2 $2^{-1-}$ mice, a mouse model for ASD. We found that in mutant mice, GH and IGF-1 mRNA levels were significantly 
higher in the pituitary gland and liver, respectively, but this increase was not paralleled by higher levels of circulating hormones. In En2 mutants, GH and IGF-1 mRNA levels were instead significantly down-regulated in the hippocampus, and this reduction was accompanied by a significant decrease of GH but not IGF-1 protein levels.

\section{En2 INTERACTS WITH IGF-1 SIGNALING IN THE MOUSE NEUROENDOCRINE AXIS}

Our results, schematically summarized in Figure 6, indicate that regulatory mechanisms controlling GH and IGF-1 mRNA

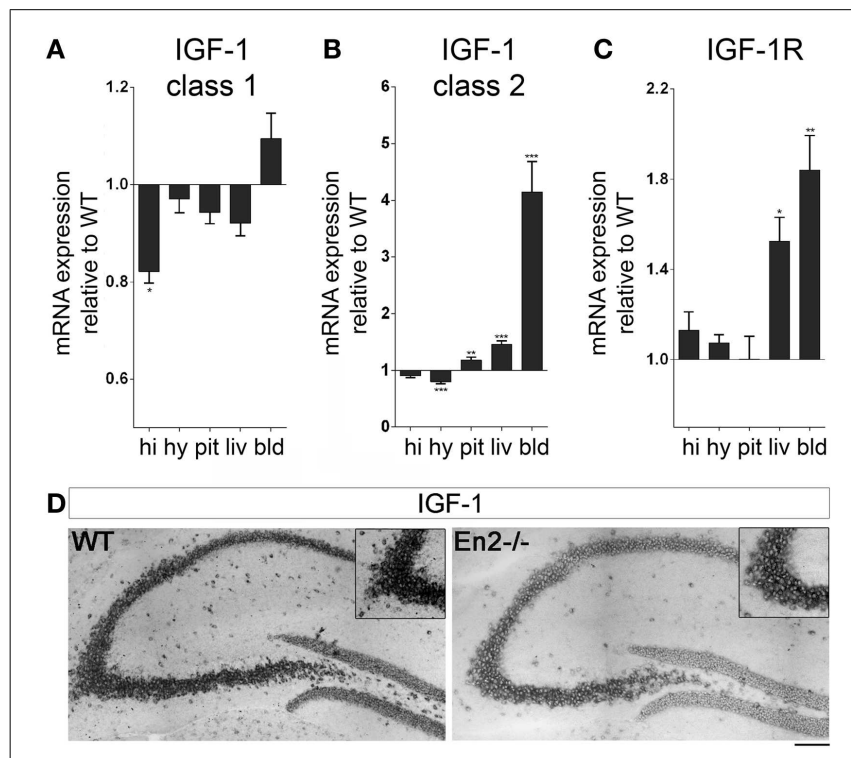

FIGURE 4 | Expression of IGF-1 and IGF-1R mRNAs in the neuroendocrine axis of WT and $E n 2^{-1-}$ mice. (A,B) Quantitative RT-PCR for IGF-1 class 1 (A) and class 2 (B) transcripts. (C) IGF-1R quantitative RT-PCR. Values are plotted as each gene/L41 comparative quantitation ratios normalized on the expression of WT (mean \pm SEM of three replicates from pools of six animals per genotype; ${ }^{*} p<0.05,{ }^{*} p<0.01$; ${ }^{* *} p<0.001$; Student's t-test, En2 ${ }^{-1-}$ vs. WT). (D) Representative pictures of in situ hybridization for IGF-1 mRNA (both transcripts) on the dorsal hippocampus from WT and En2 ${ }^{-1-}$ mice. Insets show the CA3 subfield. Scale bar: $200 \mu \mathrm{m}$ (whole hippocampi) and $125 \mu \mathrm{m}$ (insets). Abbreviations are as in Figure 1. expression in the neuroendocrine system are altered in $E n 2^{-1-}$ mice. The presence of En 2 mRNA in the hypothalamus-pituitaryliver axis (Figure 1) and that of an En2 binding site in the promoters of GH, IGF-1, and other genes of the pathway (Table 2) suggest that En2 might directly contribute to their transcriptional control. Indeed, recent studies revealed an unprecedented interaction between En2 and IGF-1 signaling. In the absence of En2, IGF-1 has a stronger mitogenic effect on cerebellar GNPs, due the increased activity of downstream effectors of IGF-1 signaling, such as S6 kinase (29). Thus, En2 appears to negatively regulate IGF-1 signaling during postnatal cerebellar development. Our results strengthen this link between En2 and IGF-1 signaling, and suggest that a direct transcriptional control of En2 onto genes belonging to the IGF-1 pathway takes place also in the brain-pituitary-liver axis.
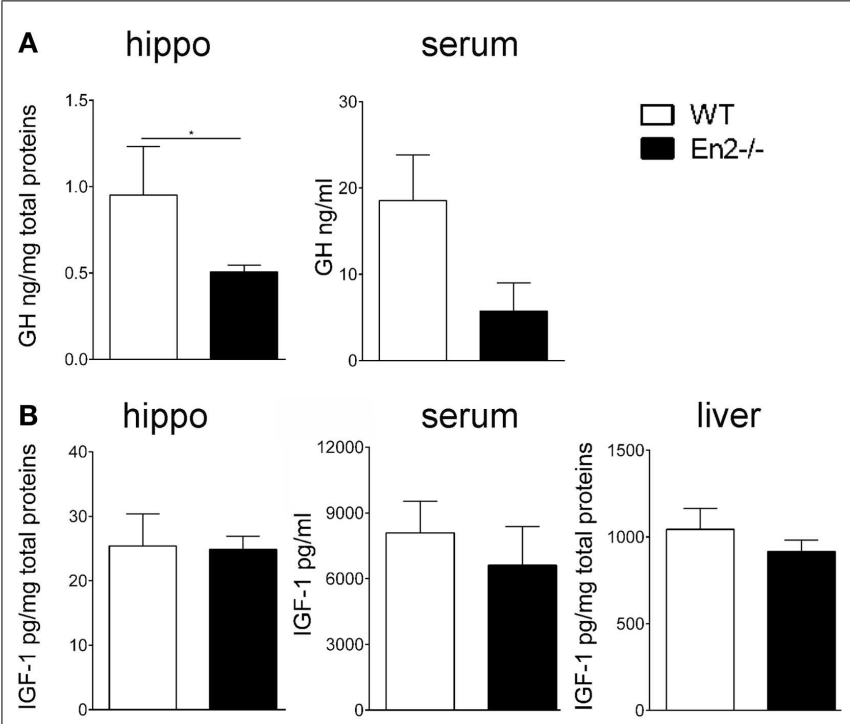

FIGURE 5 | Levels of GH and IGF-1 hormones in WT and En2 ${ }^{-/-}$mice (A,B) ELISA quantification of GH (A) and IGF-1 (B) levels in serum and hippocampal (hippo) and liver protein extracts, as indicated. Values are plotted as mean \pm SEM (five animals per genotype, in duplicate; ${ }^{*} p<0.05$,

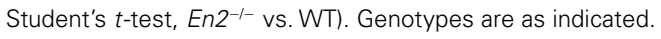

Table 3 | Summary of the expression of En2, GH, GHR, mGRF, SST, IGF-1, and IGF-1R mRNAs in the neuroendocrine axis of En2 ${ }^{-1-}$ mice, as compared to WT

\begin{tabular}{|c|c|c|c|c|c|}
\hline Gene & Hippocampus & Hypothalamus & Pituitary & Liver & Blood \\
\hline $\mathrm{GH}$ & $-98 \% * * *$ & $-97 \% * * *$ & $+72 \% * * *$ & $+376 \% * * *$ & $+87 \% *$ \\
\hline $\mathrm{GHR}$ & No difference & No difference & No difference & No difference & $+312 \% *$ \\
\hline mGRF & No difference & $+53 \% *$ & No difference & $+129 \% * *$ & $+125 \% *$ \\
\hline SST & $-10 \% *$ & $-13 \% * *$ & No difference & $+147 \% * *$ & No difference \\
\hline IGF-1 Class 1 & $-18 \% *$ & No difference & No difference & No difference & No difference \\
\hline IGF-1 Class 2 & No difference & $-19 \% * * *$ & $+16 \% * *$ & $+43 \% * * *$ & $+312 \% * * *$ \\
\hline IGF-1R & No difference & No difference & No difference & $+52 \% *$ & $+84 \% * *$ \\
\hline
\end{tabular}

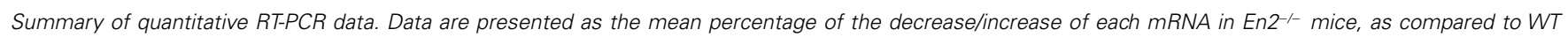
littermates.

Statistical significance: ${ }^{*} p<0.05,{ }^{*} p<0.01,{ }^{* *} p<0.001\left(E n 2^{-1-}\right.$ vs. WT). Abbreviations are as in the text. 


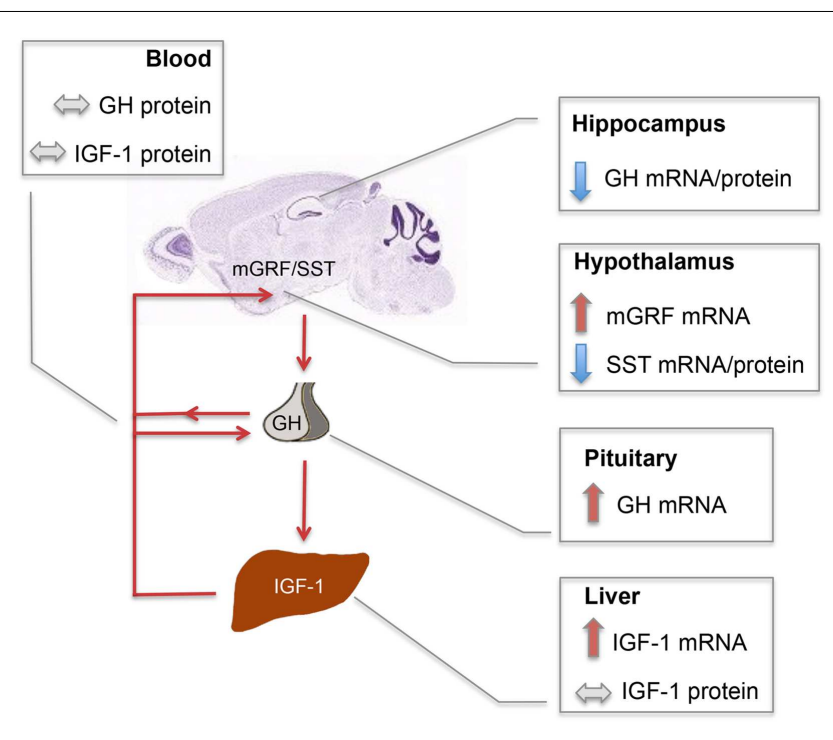

FIGURE 6 | Schematic summary of SST, mGRF, GH, and IGF-1 expression in the En2 ${ }^{-/-}$neuroendocrine axis. Red and blue arrows, respectively, indicate up- and down-regulations observed in En2 $2^{-/-}$mice as compared to WT controls. Double-arrowed gray lines indicate comparable levels between WT and En2 ${ }^{-1-}$ mice. Increased levels of GH and IGF-1

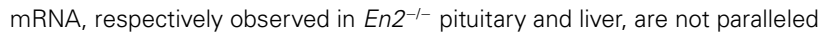
by higher levels of circulating hormones, suggesting that a complex post-translational control of GH and IGF-1 synthesis takes place in mutant mice. GH mRNA and protein levels are instead significantly down-regulated

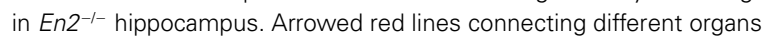
indicate the action of circulating hormones onto their target tissues. The mouse brain sagittal section is a Nissl stain taken from the Allen Mouse Brain Atlas (see text footnote 2). Abbreviations are as in the text.

\section{DIFFERENTIAL EXPRESSION OF GH AND IGF-1 mRNAs IN THE BRAIN AND PERIPHERAL TISSUES OF En2-I- MICE}

IGF-1 mRNA exists in two main isoforms, derived from alternative splicing of the two first exons contained in the $5^{\prime}$ untranslated region ( $5^{\prime}$ UTR) of the mRNA precursor. Class 1 transcripts contain exon 1, whereas class 2 transcripts contain exon 2 (33). Inclusion of exon 1 or exon 2 is mutually exclusive, resulting in different $5^{\prime}$ UTRs of the IGF-1 mRNA containing different leader sequences of the pre-pro-IGF-I peptide. In addition, transcription of class 1 and class 2 mRNAs is differently controlled; class 2 mRNA being directly regulated by GH. It is, however, important to note that both class 1 and class 2 transcripts code for the same IGF-1 mature peptide (33).

In En2 mutants, we described a differential expression of IGF1 mRNAs in different tissues of the brain-pituitary-liver axis. A significant increase of IGF-1 class 2 mRNA was detected in liver and blood, whereas IGF-1 class $1 \mathrm{mRNA}$ was significantly decreased in the En2 $2^{-/-}$hippocampus (Figure 4). The negative regulation exerted by En 2 onto IGF-1 signaling in the cerebellum (29), therefore, appears to occur also at a transcriptional level in the hippocampus.

Interestingly, En $2^{-/-}$mice showed increased hypothalamic levels of mGRF and decreased levels of somatostatin (SST), the two hormones controlling GH synthesis. Previous studies from our laboratory already showed that loss of En2 results in lower levels of SST mRNA and protein in the hippocampus and cerebral cortex. Here, we extended this observation, demonstrating that lower levels of SST are also present in the En2 $2^{-1-}$ hypothalamus; hypothalamic levels of mGRF mRNA were instead increased in mutant mice (Figure 3). Deregulation of hypothalamic hormones controlling GH synthesis might result in increased levels of circulating GH and IGF-1 in mutant mice. However, we showed that serum levels of both hormones, as well as IGF1 levels in liver did not differ between WT and En $2^{-/-}$mice (Figure 5). Indeed, behavioral studies did not reveal gross weight and growth abnormalities in En2 mutants (23), suggesting that differences in GH and IGF-1 mRNA levels might be blunted at the protein level, via multiple post-transcriptional control mechanisms. Indeed, a tight dependence of IGF-1 translation via several miRNA binding site in the $3^{\prime}$ UTR of the transcripts has been described (33, 35).

\section{FUNCTIONAL CONSEQUENCES OF REDUCED GH LEVELS IN THE En2 ${ }^{-/-}$ HIPPOCAMPUS}

The decreased expression of GH mRNA and protein observed in the En2 $2^{-/-}$hippocampus might contribute to learning deficits observed in En2 mutants. Indeed, En2 ${ }^{-1-}$ mice show impaired learning in hippocampal-dependent tasks, such as the Morris water maze and contextual fear conditioning (22, 23). Profound effects on cognitive function have been demonstrated for GH. GH-deficient spontaneous dwarf rats display marked deficits in hippocampus-dependent spatial learning and memory, accompanied by an imbalance in hippocampal glutamatergic/GABAergic synapses and neurogenesis (36). Chronic stress, which is known to affect hippocampal function, also reduces hippocampal GH levels (37). Restoration of normal GH levels in the hippocampus (obtained via viral-mediated gene transfer) is able to reverse stress-dependent behavioral impairment, as tested by hippocampus-dependent tasks (contextual fear conditioning) (37). Taken together, these results indicate that reduced levels of hippocampal GH detected in En2 $2^{-/-}$mice might contribute to hippocampal dysfunction observed in these mutants.

\section{CONCLUSION}

To our knowledge, this is the first demonstration that brain GH levels are reduced in a mouse model of ASD. Considering the important role of $\mathrm{GH}$ on cognitive functions, our data strengthen the notion that reduced expression of GH in the hippocampus may be implicated in learning disabilities associated to ASD.

\section{AUTHOR CONTRIBUTIONS}

Giovanni Provenzano and Elena Clementi equally contributed to this study. Giovanni Provenzano designed and performed experiments, analyzed data and wrote the paper. Elena Clementi designed and performed experiments, analyzed data. Sacha Genovesi, Manuela Scali, Prem Prakash Tripathi, and Paola Sgadò performed experiments. Yuri Bozzi provided funding, conceived the study, analyzed data and wrote the paper.

\section{ACKNOWLEDGMENTS}

We are grateful to the technical/administrative staff of the Centre for Integrative Biology (University of Trento). We thank 
Prof. Emiliana Borrelli (University of California, Irvine, CA, USA) for the kind gift of GH and mGRF cDNAs. This work was funded by grants from the Italian Ministry of University and Research (PRIN 2008 grant 200894SYW2_002 and PRIN 2010-11 grant 2010N8PBAA_002) and the University of Trento (CIBIO start-up) to Yuri Bozzi. Paola Sgadò is supported by Provincia Autonoma di Trento (Italy) under the Marie Curie-People cofunding action of the European Community.

\section{REFERENCES}

1. Llorens-Martín M, Torres-Alemán I, Trejo JL. Mechanisms mediating brain plasticity: IGF1 and adult hippocampal neurogenesis. Neuroscientist (2009) 15:134-48. doi:10.1177/1073858408331371

2. Sale A, Cenni MC, Ciucci F, Putignano E, Chierzi S, Maffei L. Maternal enrichment during pregnancy accelerates retinal development of the fetus. PLoS One (2007) 2:e1160. doi:10.1371/journal.pone.0001160

3. Sale A, Berardi N, Maffei L. Enrich the environment to empower the brain. Trends Neurosci (2009) 32:233-9. doi:10.1016/j.tins.2008.12.004

4. Guzzetta A, Baldini S, Bancale A, Baroncelli L, Ciucci F, Ghirri P, et al. Massage accelerates brain development and the maturation of visual function. J Neurosci (2009) 29:6042-51. doi:10.1523/JNEUROSCI.5548-08.2009

5. Torres-Aleman I. Toward a comprehensive neurobiology of IGF-I. Dev Neurobiol (2010) 70:384-96. doi:10.1002/dneu.20778

6. Trejo JL, Llorens-Martín MV, Torres-Alemán I. The effects of exercise on spatial learning and anxiety-like behavior are mediated by an IGF-I-dependent mechanism related to hippocampal neurogenesis. Mol Cell Neurosci (2008) 37:402-11. doi:10.1016/j.mcn.2007.10.016

7. Llorens-Martín M, Torres-Alemán I, Trejo JL. Exercise modulates insulin-like growth factor 1-dependent and -independent effects on adult hippocampal neurogenesis and behaviour. Mol Cell Neurosci (2010) 44:109-17. doi:10.1016/j. mcn.2010.02.006

8. Trejo JL, Piriz J, Llorens-Martín MV, Fernandez AM, Bolós M, LeRoith D, et al. Central actions of liver-derived insulin-like growth factor I underlying its pro-cognitive effects. Mol Psychiatry (2007) 12:1118-28. doi:10.1038/sj.mp. 4002076

9. Mills JL, Hediger ML, Molloy CA, Chrousos GP, Manning-Courtney P, Yu KF, et al. Elevated levels of growth-related hormones in autism and autism spectrum disorder. Clin Endocrinol (2007) 67:230-7. doi:10.1111/j.1365-2265.2007. 02868.x

10. Vanhala R, Turpeinen U, Riikonen R. Low levels of insulin-like growth factor-I in cerebrospinal fluid in children with autism. Dev Med Child Neurol (2001) 43:614-6. doi:10.1017/S0012162201001116

11. Riikonen R, Makkonen I, Vanhala R, Turpeinen U, Kuikka J, Kokki H. Cerebrospinal fluid insulin-like growth factors IGF-1 and IGF-2 in infantile autism. Dev Med Child Neurol (2006) 48:751-5. doi:10.1017/S0012162206001605

12. Chen J, Alberts I, Li X. Dysregulation of the IGF-I/PI3K/AKT/mTOR signaling pathway in autism spectrum disorders. Int J Dev Neurosci (2014) 35C:35-41. doi:10.1016/j.ijdevneu.2014.03.006

13. Shcheglovitov A, Shcheglovitova O, Yazawa M, Portmann T, Shu R, Sebastiano V, et al. SHANK3 and IGF1 restore synaptic deficits in neurons from 22q13 deletion syndrome patients. Nature (2013) 503:267-71. doi:10.1038/nature12618

14. Tropea D, Giacometti E, Wilson NR, Beard C, McCurry C, Fu DD, et al. Partial reversal of Rett Syndrome-like symptoms in MeCP2 mutant mice. Proc Natl Acad Sci U S A (2009) 106:2029-34. doi:10.1073/pnas.0812394106

15. Bozdagi O, Tavassoli T, Buxbaum JD. Insulin-like growth factor-1 rescues synaptic and motor deficits in a mouse model of autism and developmental delay. $\mathrm{Mol}$ Autism (2013) 4:9. doi:10.1186/2040-2392-4-9

16. Nyberg F, Hallberg M. Growth hormone and cognitive function. Nat Rev Endocrinol (2013) 9:357-65. doi:10.1038/nrendo.2013.78

17. Iwata K, Matsuzaki H, Miyachi T, Shimmura C, Suda S, Tsuchiya KJ, et al. Investigation of the serum levels of anterior pituitary hormones in male children with autism. Mol Autism (2011) 2:16. doi:10.1186/2040-2392-2-16

18. Benayed R, Choi J, Matteson PG, Gharani N, Kamdar S, Brzustowicz LM, et al. Autism-associated haplotype affects the regulation of the homeobox gene, ENGRAILED 2. Biol Psychiatry (2009) 66:911-7. doi:10.1016/j.biopsych.2009. 05.027
19. James SJ, Shpyleva S, Melnyk S, Pavliv O, Pogribny IP. Complex epigenetic regulation of Engrailed-2 (EN-2) homeobox gene in the autism cerebellum. Transl Psychiatry (2013) 3:e232. doi:10.1038/tp.2013.8

20. Choi J, Ababon MR, Soliman M, Lin Y, Brzustowicz LM, Matteson PG, et al. Autism associated gene, Engrailed 2, and flanking gene levels are altered in post-mortem cerebellum. PLoS One (2014) 9:e87208. doi:10.1371/journal.pone. 0087208

21. Joyner AL, Herrup K, Auerbach BA, Davis CA, Rossant J. Subtle cerebellar phenotype in mice homozygous for a targeted deletion of the En-2 homeobox. Science (1991) 251:1239-43. doi:10.1126/science.1672471

22. Cheh MA, Millonig JH, Roselli LM, Ming X, Jacobsen E, Kamdar S, et al. En2 knockout mice display neurobehavioral and neurochemical alterations relevant to autism spectrum disorder. Brain Res (2006) 1116:166-76. doi:10.1016/ j.brainres.2006.07.086

23. Brielmaier J, Matteson PG, Silvermanm JL, Senerth JM, Kelly S, Genestine M, et al. Autism-relevant social abnormalities and cognitive deficits in Engrailed-2 knockout mice. PLoS One (2012) 7:e40914. doi:10.1371/journal.pone.0040914

24. Brielmaier J, Senerth JM, Silverman JL, Matteson PG, Millonig JH, DiCiccoBloom E, et al. Chronic desipramine treatment rescues depression-related, social and cognitive deficits in Engrailed-2 knockout mice. Genes Brain Behav (2014) 13:286-98. doi:10.1111/gbb.12115

25. Tripathi PP, Sgadò P, Scali M, Viaggi C, Casarosa S, Simon HH, et al. Increased susceptibility to kainic acid-induced seizures in Engrailed-2 knockout mice. Neuroscience (2009) 159:842-9. doi:10.1016/j.neuroscience.2009.01.007

26. Kuemerle B, Zanjani H, Joyner A, Herrup K. Pattern deformities and cell loss in Engrailed-2 mutant mice suggest two separate patterning events during cerebellar development. J Neurosci (1997) 17:7881-9.

27. Sgadò P, Genovesi S, Kalinovsky A, Zunino G, Macchi F, Allegra M, et al. Loss of GABAergic neurons in the hippocampus and cerebral cortex of Engrailed-2 null mutant mice: implications for autism spectrum disorders. Exp Neurol (2013) 247:496-505. doi:10.1016/j.expneurol.2013.01.021

28. Sgadò P, Provenzano G, Dassi E, Adami V, Zunino G, Genovesi S, et al. Transcriptome profiling in Engrailed-2 mutant mice reveals common molecular pathways associated with autism spectrum disorders. Mol Autism (2013) 4:51. doi:10.1186/2040-2392-4-51

29. Rossman IT, Lin L, Morgan KM, Digiovine M, Van Buskirk EK, Kamdar S, et al. Engrailed 2 modulates cerebellar granule neuron precursor proliferation, differentiation and insulin-like growth factor 1 signaling during postnatal development. Mol Autism (2014) 5:9. doi:10.1186/2040-2392-5-9

30. Saiardi A, Bozzi Y, Baik JH, Borrelli E. Antiproliferative role of dopamine: loss of $\mathrm{D} 2$ receptors causes hormonal dysfunction and pituitary hyperplasia. Neuron (1997) 19:115-26. doi:10.1016/S0896-6273(00)80352-9

31. Park OK, Gugneja S, Mayo KE. Gonadotropin-releasing hormone gene expression during the rat estrous cycle: effects of pentobarbital and ovarian steroids. Endocrinology (1990) 127:365-72. doi:10.1210/endo-127-1-365

32. Rotwein P. Two insulin-like growth factor I messenger RNAs are expressed in human liver. Proc Natl Acad Sci U S A (1986) 83:77-81. doi:10.1073/pnas.83.1.77

33. Oberbauer AM. The regulation of IGF-1 gene transcription and splicing during development and aging. Front Endocrinol (2013) 4:39. doi:10.3389/fendo.2013. 00039

34. Mathews LS, Norstedt G, Palmiter RD. Regulation of insulin-like growth factor I gene expression by growth hormone. Proc Natl Acad Sci U S A (1986) 83:9343-7. doi:10.1073/pnas.83.24.9343

35. Xie X, Lu J, Kulbokas E, Golub TR, Mootha V, Lindblad-Toh K, et al. Systematic discovery of regulatory motifs in human promoters and 3' UTRs by comparison of several mammals. Nature (2005) 434:338-45. doi:10.1038/nature03441

36. Li E, Kim DH, Cai M, Lee S, Kim Y, Lim E, et al. Hippocampus-dependent spatial learning and memory are impaired in growth hormone-deficient spontaneous dwarf rats. Endocr J (2011) 58:257-67. doi:10.1507/endocrj.K11E-006

37. Vander Weele CM, Saenz C, Yao J, Correia SS, Goosens KA. Restoration of hippocampal growth hormone reverses stress-induced hippocampal impairment. Front Behav Neurosci (2013) 7:66. doi:10.3389/fnbeh.2013.00066

Conflict of Interest Statement: The Reviewer, Dr Alessandro Sale, declares that despite being affiliated with the same institution as author Dr Manuela Scali and having collaborated with her in the past, the review process was handled objectively and no conflict of interest exists. The authors declare that the research was conducted in the absence of any commercial or financial relationships that could be construed as a potential conflict of interest. 
Received: 27 May 2014; accepted: 18 August 2014; published online: 01 September 2014. Citation: Provenzano G, Clementi E, Genovesi S, Scali M, Tripathi PP, Sgadò P and Bozzi Y (2014) GH dysfunction in Engrailed-2 knockout mice, a model for autism spectrum disorders. Front. Pediatr. 2:92. doi: 10.3389/fped.2014.00092

This article was submitted to Child and Neurodevelopmental Psychiatry, a section of the journal Frontiers in Pediatrics.
Copyright (C) 2014 Provenzano, Clementi, Genovesi, Scali, Tripathi, Sgadò and Bozzi. This is an open-access article distributed under the terms of the Creative Commons Attribution License (CC BY). The use, distribution or reproduction in other forums is permitted, provided the original author(s) or licensor are credited and that the original publication in this journal is cited, in accordance with accepted academic practice. No use, distribution or reproduction is permitted which does not comply with these terms. 